\title{
The Association Of Laparoscopic Surgeries And Postoperative Renal Injury In Noncardiac Surgery- a Retrospective Analysis
}

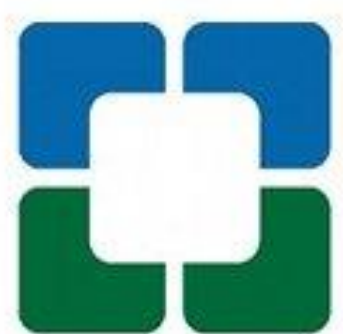

Cleveland Clinic

\author{
Barak Cohen, M.D.', Steve M. Leung, M.D.1, Hani A. Essber, M.D.1, Amanda Naylor', \\ Amanda Artis, M.S. ${ }^{2}$, Alparslan Turan, M.D. ${ }^{1,}$ Daniel I Sessler, M.D. ${ }^{1}$,Kurt Ruetzler, M.D. ${ }^{1}$ \\ ${ }^{1}$ Department of Outcomes Research, Cleveland Clinic - Cleveland, USA \\ ${ }^{2}$ Quantitative Health Sciences, Cleveland Clinic - Cleveland, USA
}

\section{Introduction and Hypothesis}

Laparoscopic procedures are common and can enhance recovery, decrease postoperative pain, and shorten hospital length of stay (LOS). Nevertheless, increased intra-abdominal pressure (IAP) is associated with decreased renal blood flow, renal hypoxia and acute kidney injury. When combined with the Trendelenburg position, renal function may further deteriorate due to increased central venous pressure. The effects of intraoperative increase in IAP combined with Trendelenburg position on post-operative renal outcomes are unknown. Hypothesis: Combination of laparoscopic approach and Trendelenburg position during adult colorectal surgery is associated primarily with decrease in estimated glomerular filtration rate (eGFR) and secondarily with increased incidence of acute kidney injury (AKI), compared to laparotomy without Trendelenburg position.

Patients and Methods

In this retrospective cohort study, adult patients undergoing laparoscopic colorectal surgery in Trendelenburg position in the Cleveland Clinic were propensity-matched to patients undergoing similar procedures in an open approach. Patients with preoperative renal failure or critical illness were excluded. Matching considered preoperative potential confounders and was defined appropriate when the absolute standardized difference was $<0.1$. When matching was not achieved, multilinear regression was used. The primary outcome was eGFR, calculated according to the Chronic Kidney Disease Epidemiology collaboration (CKD-EPI), and secondary outcomes were AKI (defined by the Acute Kidney Injury Network - AKIN, modified to 7 postoperative days) and hospital LOS.

\section{Results}

Of 7,357 eligible patients, 1,846 laparoscopic cases with Trendelenburg were matched to 1,846 control cases. All considered confounders were well balanced aside from one that was adjusted by multilinear regression. No difference was found in eGFR reduction (difference between the groups of $1.12 \mathrm{~mL} / \mathrm{min} / 1.73 \mathrm{~m} 2[95 \% \mathrm{Cl}-0.42$, 2.67] $p=0.155$ ). The relative risk of $A K I$ in the laparoscopic group was $0.7[95 \% \mathrm{Cl} 0.55,0.90, \mathrm{p}=0.006]$. LOS was also shorter in the laparoscopic group, with 1.21 times higher chance of earlier discharge than the open surgery group $[95 \% \mathrm{Cl} 1.14,1.29, p<0.001]$.

\section{Conclusion}

Despite compelling potential mechanisms, laparoscopic approach with Trendelenburg position in adult colorectal surgeries did not alter postoperative eGFR and actually decreased the risk of postoperative acute kidney injury compared to open colorectal surgeries with no Trendelenburg position. It also shortened length of stay after these procedures.

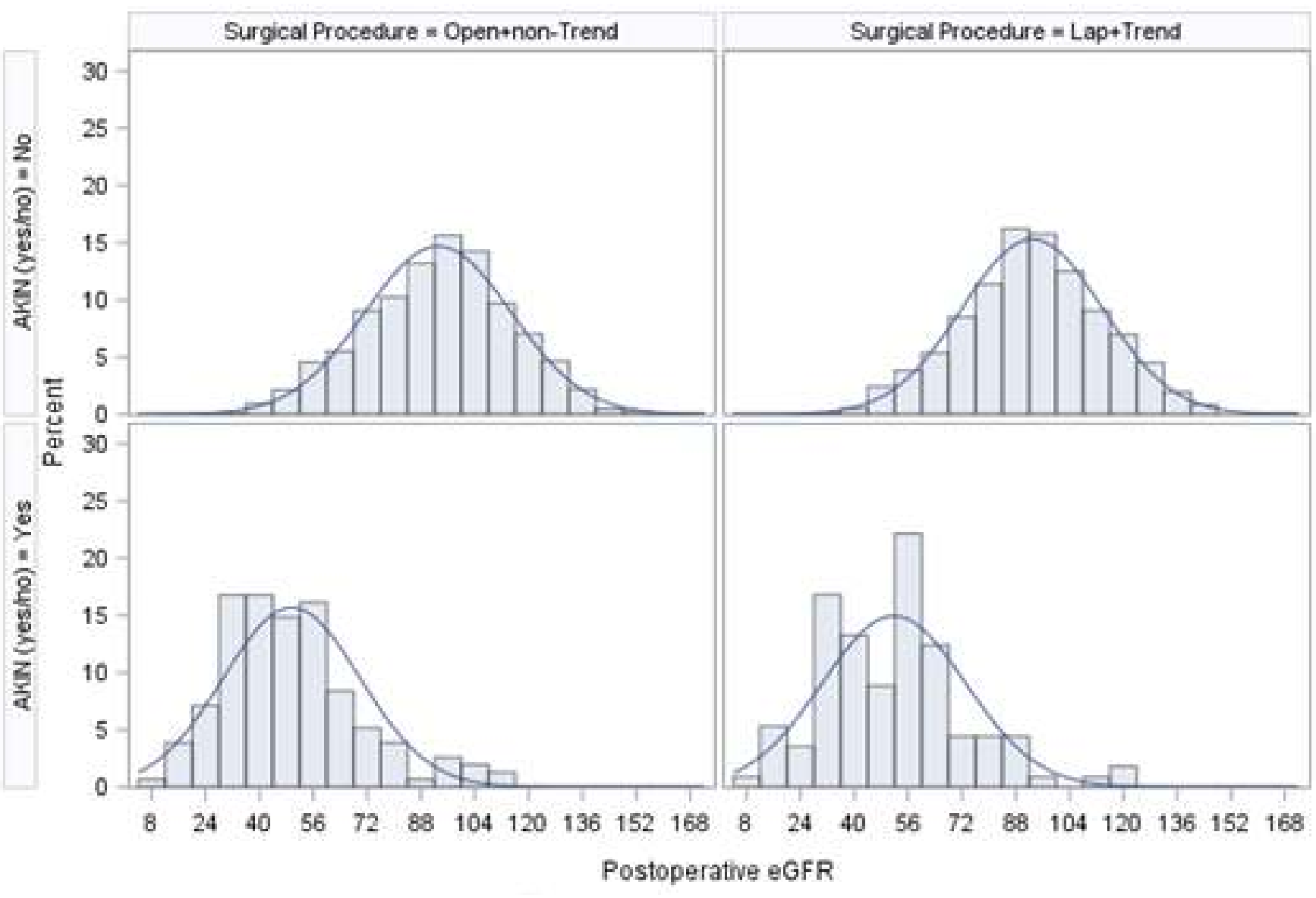

Figure 1: The distributions of eGFR are similar between the two procedures, which is why we don't see a significant difference between laparoscopic approach with Trendelenburg position and eGFR.

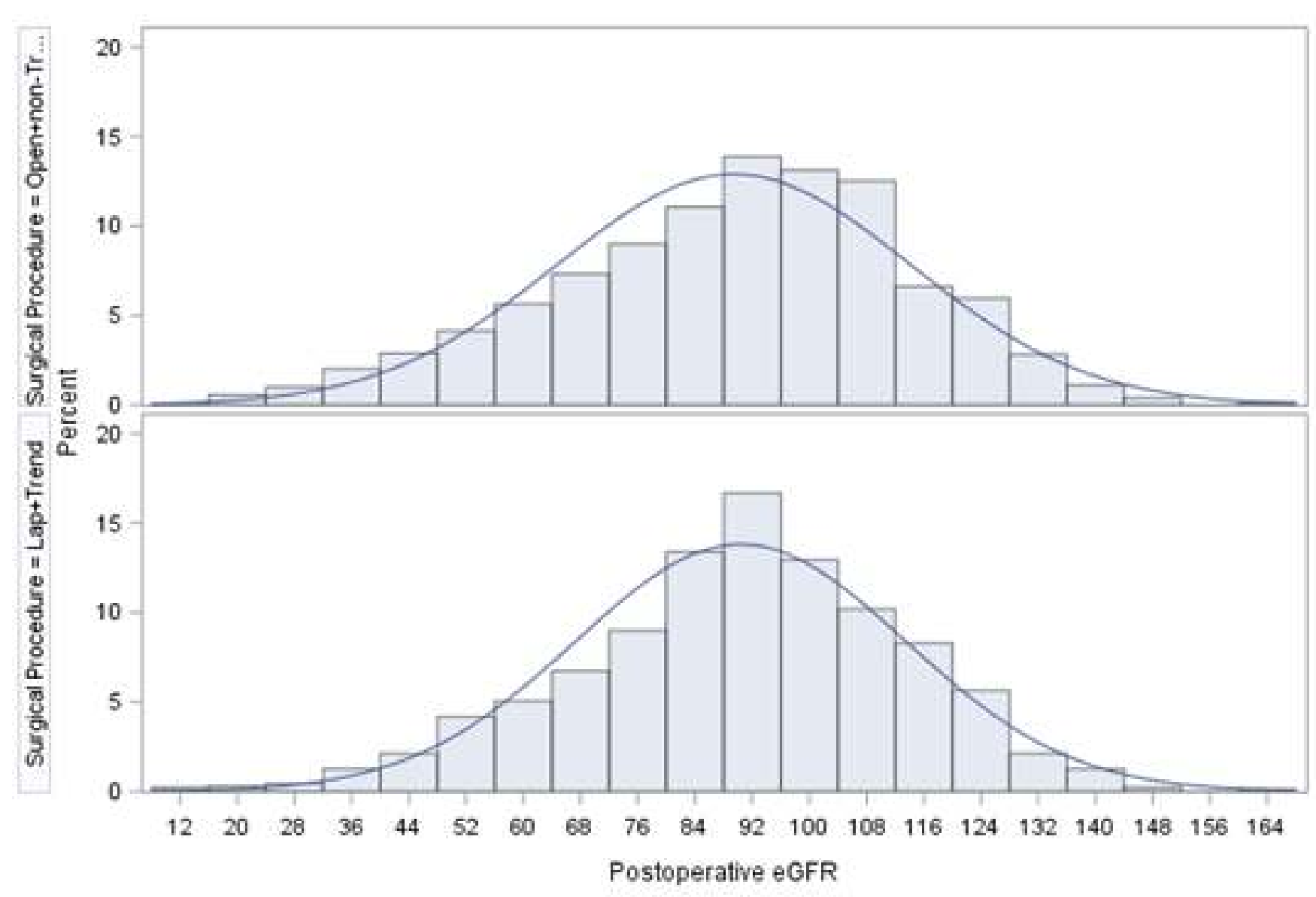

Figure 2: eGFR by procedure where no drastic difference in the distributions for eGFR is apparent between the two groups. 